\title{
Uses of Transmission Electron Microscope in Microscopy and its Advantages and Disadvantages
}

\author{
Priyanka $^{1}$ and Om Prakash Choudhary ${ }^{2 *}$
}

${ }^{1}$ Department of Veterinary Microbiology, College of Veterinary and Animal Sciences, Rajasthan University of Veterinary and Animal Sciences, Bikaner-334001, Rajasthan, India

${ }^{2}$ Department of Veterinary Anatomy and Histology, College of Veterinary Sciences and Animal Husbandry, Central Agricultural University, Selesih, Aizawl- 796015, Mizoram, India

*Corresponding author

\section{A B S T R A C T}

\begin{tabular}{|l|}
\hline Ke y w or d s \\
TEM, Electrons, de \\
Broglie wavelength, \\
Nanotechnology, \\
CCD camera
\end{tabular}

Transmission electron microscopy (TEM) is a microscopic technique in which a beam of electrons is transmitted through an ultra-thin specimen, interacting with the specimen as it passes through. An image is formed by the interaction of the electrons transmitted through the specimen; the image is magnified and focused onto an imaging device, such as a fluorescent screen or on a layer of photographic film, or to be detected by a sensor such as a CCD camera. TEMs are capable of imaging at a significantly higher resolution than light microscopes, owing to the small de Broglie wavelength of electrons. This enables the instrument's user to examine fine detail-even as small as a single column of atoms, which is thousands of times smaller than the smallest resolvable object in a light microscope. TEM forms a major analytical tool in physical as well as biological sciences. it finds application in cancer research, virology, materials science as well as pollution, nanotechnology, and semiconductor research etc.

\section{Introduction}

Ernst Abbe originally proposed that the ability to resolve the detail in an object was limited approximately by the wavelength of the light used in imaging, which limits the resolution of an optical microscope to a few hundred nanometers. Evolutions development of the ultraviolet (UV) microscopes, led by Köhler and Rohr, allowed for an increase in resolving power of about a factor of two (Encyclopcedia Britannica Online). However this required more expensive quartz optical components, due to the absorption of UV by glass. At this point it was believed that obtaining an image with sub-micrometer information was simply impossible due to this wavelength constraint (Ernst Ruska).

It had earlier been recognized by Plücker in 1858 that the deflection of "cathode rays" (electrons) was possible by the use of magnetic fields (Plücker, 1858). This effect had been utilized to build primitive cathode ray oscilloscopes (CROs) as early as 1897 by Ferdinand Braun, intended as a measurement device. (nobelprize.org) Indeed in 1891 it was recognized by Riecke that the cathode rays 
could be focused by these magnetic fields, allowing for simple lens designs. Later this theory was extended by Hans Busch in his work published in 1926, who showed that the lens maker's equation, under appropriate assumptions, could be applicable to electrons. (nobelprize.org)

In 1928, at the Technological University of Berlin Adolf Matthias, Professor of High voltage Technology and Electrical Installations, appointed Max Knoll to lead a team of researchers to advance the CRO design. The team consisted of several Ph.D. students including Ernst Ruska and Bodo von Borries.

This team of researchers concerned themselves with lens design and CRO column placement, which they attempted to optimize to obtain the parameters which could to allow for construction of better CROs, as well as the development of electron optical components which could be used to generate low magnification (nearly 1:1) images. In 1931, the group successfully generated magnified images of mesh grids placed over the anode aperture. The device used two magnetic lenses to achieve higher magnifications, arguably the first electron microscope. In that same year, Reinhold Rudenberg, the scientific director of the Siemens Company, had patented an electrostatic lens electron microscope (Rudenberg, Reinhold, 1931).

A Transmission Electron Microscope (TEM) utilizes energetic electrons to provide morphologic, compositional and crystallographic information on samples. At a maximum potential magnification of 1 nanometer, TEMs are the most powerful microscopes. TEMs produce high-resolution, two-dimensional images, allowing for a wide range of educational, science and industry related applications. At smaller magnifications, TEM image contrast is due to absorption of electrons in the material, due to the thickness and composition of the material. At higher magnifications complex wave interactions modulate the intensity of the image, requiring expert analysis of observed images. Alternate modes of uses of the TEM allows the observation of modulations in chemical identity, crystal orientation, electronic structure and sample induced electron phase shift as well as the regular absorption based imaging.

\section{Components of TEM}

An electron source: From the top down, the TEM consists of an emission source, which may be a tungsten filament, or a lanthanum hexaboride $\left(\mathrm{LaB}_{6}\right)$ source (Egerton, 2005). For tungsten, this will be of the form of either a hairpin-style filament, or a small spike-shaped filament. $\mathrm{LaB}_{6}$ (Lanthanum hexaboride) sources utilize small single crystals. By connecting this gun to a high voltage source (typically $\sim 100-300 \mathrm{kV}$ ) the gun will, given sufficient current, and begins to emit electrons either by thermionic or field electron emission into the vacuum. This extraction is usually aided by the use of a Wehnelt cylinder. Once extracted, the upper lenses of the TEM allow formation of the electron probe to the desired size and location for later interaction with the sample (Rose, 2008).

Thermionic Gun

Electron beam

Electromagnetic lenses: Electron lenses are designed to act in a manner emulating that of an optical lens, by focusing parallel rays at some constant focal length. Lenses may operate electrostatically or magnetically. The majority of electron lenses for TEM utilize electromagnetic coils to generate a convex lens. For these lenses the field produced for the lens must be radially symmetrical, as deviation from the radial symmetry of the 
magnetic lens causes aberrations such as astigmatism, and worsens spherical and chromatic aberration. Electron lenses are manufactured from iron, iron-cobalt or nickel cobalt alloys, such as Permalloy (Orloff, 1997). These are selected for their magnetic properties, such as magnetic saturation, hysteresis and permeability.

Vacuum chamber

Two Condensers

Sample stage

Phosphor or fluorescent screen

Computer for imaging

A Transmission Electron Microscope functions under the same basic principles as an optical microscope. In a TEM, electrons replace photons, electromagnetic lenses replace glass lenses and images are viewed on a screen rather than through an eyepiece.

\section{TEM imaging}

\section{A Transmission Electron Microscope} produces a high-resolution, black and white image from the interaction that takes place between prepared samples and energetic electrons in the vacuum chamber. Air needs to be pumped out of the vacuum chamber, creating a space where electrons are able to move. The electrons then pass through multiple electromagnetic lenses. These solenoids are tubes with coil wrapped around them. The beam passes through the solenoids, down the column, makes contact with the screen where the electrons are converted to light and form an image. The image can be manipulated by adjusting the voltage of the gun to accelerate or decrease the speed of electrons as well as changing the electromagnetic wavelength via the solenoids. The coils focus images onto a screen or photographic plate. During transmission, the speed of electrons is directly correlated with the electron wavelength; the faster the electrons move, with shorter wavelength and the greater the quality and detail of the image.

The lighter areas of the image represent the places where a greater number of electrons were able to pass through the sample and the darker areas reflect the dense areas of the object. These differences provide information on the structure, texture, shape and size of the sample. To obtain a TEM analysis, samples need to have certain properties. They need to be sliced thin enough for electrons to pass through, a property known as electron transparency. Samples need to be able to withstand the vacuum chamber and often require special preparation before viewing.

The steps of sample preparation include dehydration, sputter coating of non-conductive materials, cryo-fixation, embedding in hard resins, sectioning and staining for contrast protection of samples.

\section{TEM applications}

A Transmission Electron Microscope is ideal for a number of diverse fields such as life sciences, nanotechnology, medical, biological and material research, forensic analysis, gemology and metallurgy as well as industry and education.

TEMs provide topographical, morphological, compositional and crystalline information. The images allow the researchers to view samples on a molecular level, making it possible to analyze structure and texture.

This information is useful in the study of crystals and metals, in addition to industrial applications.

TEMs can be used in semiconductor analysis and the production and manufacture of computer and silicon chips. 
Fig.1 Transmission electron microscope (JEOL-JEM 1011, EM Laboratory, G.B. Pant University of Agriculture and Technology, Pantnagar) showing High tension cable (HTC), Filament chamber (FiC), Vacuum chamber ( $\mathrm{VaC})$, Objective aperture (ObA), Specimen chamber $(\mathrm{SpC})$, Condenser aperture (CoA), Projector aperture (PrA), Charge-coupled device Camera (CCDC), Optical binoculars (OpBi), Floroscent screen (FloS) and Focusing control (FoC)

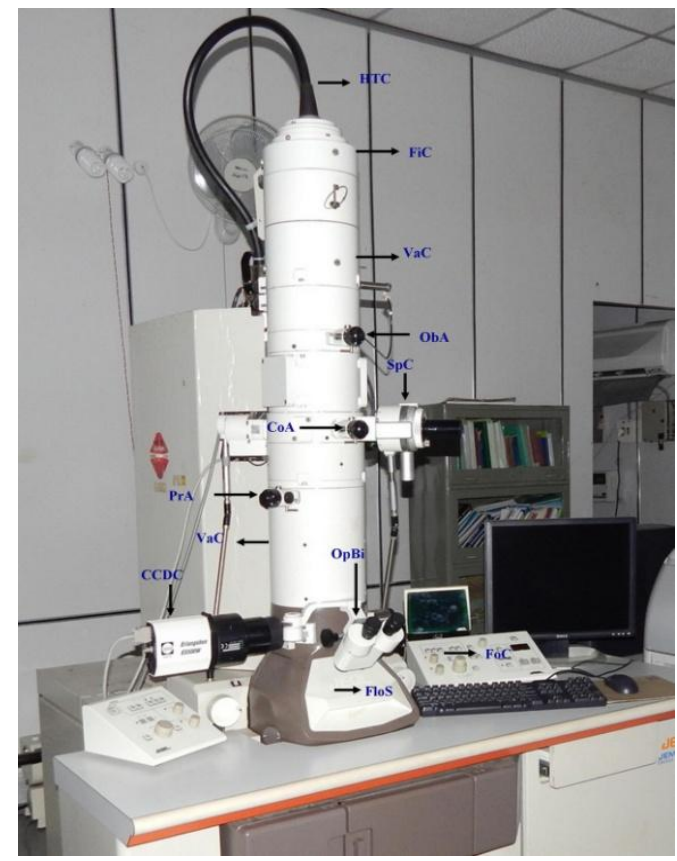

Technology based companies use TEMs to identify flaws, fractures and damages to micro-sized objects; this data can help fix problems and/or help to make a more durable, efficient product.

Colleges and universities can utilize TEMs for research and studies.

Although electron microscopes require specialized training, students can assist professors and learn TEM techniques.

Students will have the opportunity to observe a nano-sized world in incredible depth and detail (Fig. 1).

\section{Advantages}

A Transmission Electron Microscope is an impressive instrument with a number of advantages such as:
TEMs offer the most powerful magnification, potentially over one million times or more

TEMs have a wide-range of applications and can be utilized in a variety of different scientific, educational and industrial fields

TEMs provide information on the element and compound structure

Images are high-quality and detailed

TEMs are able to yield an insight of the surface features, shape, size and structure

They are easy to operate with proper training

\section{Disadvantages}

Some cons of electron microscopes include:

TEMs are large and very expensive 
Laborious sample preparation

Potential artifacts from sample preparation

Operation and analysis requires special training

Samples are limited to those that are electron transparent, able to tolerate the vacuum chamber and small enough to fit in the chamber

TEMs require special housing and maintenance facilities.

Images are black and white

Electron microscopes are sensitive to vibration and electromagnetic fields and must be housed in an area that isolates them from possible exposure. A Transmission Electron Microscope requires constant upkeep including, maintaining the voltage, current in the electromagnetic coils and cooling water.

\section{References}

Ferdinand B. (1909). The Nobel Prize in Physics. Biography. nobelprize.org.

The Nobel Prize in Physics 1986, Perspectives - Life through a Lens. nobelprize.org.
Egerton, R. (2005). Physical principles of electron microscopy. Springer. ISBN 0387-25800-0.

Ernst Ruska, translation by $\mathrm{T}$ Mulvey. The Early Development of Electron Lenses and Electron Microscopy. ISBN 37776-0364-3.

Orloff, J. (1997). Handbook of Electron Optics. CRC-press. ISBN 0-8493-25137.

Plücker, J. (1858). Über die Einwirkung des Magneten auf die elektrischen Entladungen in verdünnten Gasen [On the effect of a magnet on the electric discharge in rarified gases]. Poggendorffs Annalen der Physik und Chemie 103: 88-106.

Rose, H. H. (2008). Optics of highperformance electron Microscopes. Science and Technology of Advanced Materials (free download review on electron optics) 9: 014107.

Rudenberg, R. (1931). Configuration for the enlarged imaging of objects by electron beams. Patent DE906737.

Ultraviolet microscope. (2010). In Encyclopaedia Britannica. Retrieved November 20, 2010, from the Encyclopædia Britannica Online.

\section{How to cite this article:}

Priyanka and Om Prakash Choudhary. 2018. Uses of Transmission Electron Microscope in Microscopy and its Advantages and Disadvantages. Int.J.Curr.Microbiol.App.Sci. 7(05): 743747. doi: https://doi.org/10.20546/ijcmas.2018.705.090 